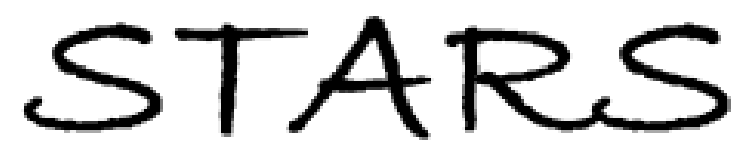

University of Central Florida

STARS

$1-1-1986$

\title{
A Model For Vocal Fold Vibratory Motion, Contact Area, And The Electroglottogram
}

D. G. Childers

D. M. Hicks

G. P. Moore

Y. A. Alsaka

University of Central Florida

Find similar works at: https://stars.library.ucf.edu/facultybib1980

University of Central Florida Libraries http://library.ucf.edu

This Article is brought to you for free and open access by the Faculty Bibliography at STARS. It has been accepted for inclusion in Faculty Bibliography 1980s by an authorized administrator of STARS. For more information, please contact STARS@ucf.edu.

\section{Recommended Citation}

Childers, D. G.; Hicks, D. M.; Moore, G. P.; and Alsaka, Y. A., "A Model For Vocal Fold Vibratory Motion, Contact Area, And The Electroglottogram" (1986). Faculty Bibliography 1980s. 479.

https://stars.library.ucf.edu/facultybib1980/479

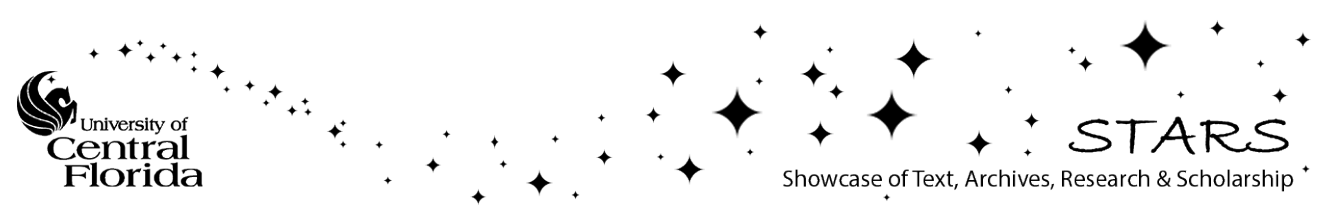




\section{A model for vocal fold vibratory motion, contact area, and the electroglottogram}

D. G. Childers, D. M. Hicks, G. P. Moore, and Y. A. Alsaka

Citation: The Journal of the Acoustical Society of America 80, 1309 (1986); doi: 10.1121/1.394382

View online: https://doi.org/10.1121/1.394382

View Table of Contents: https://asa.scitation.org/toc/jas/80/5

Published by the Acoustical Society of America

\section{ARTICLES YOU MAY BE INTERESTED IN}

A finite-element model of vocal-fold vibration

The Journal of the Acoustical Society of America 108, 3003 (2000); https://doi.org/10.1121/1.1324678

Parameterization of the glottal area, glottal flow, and vocal fold contact area

The Journal of the Acoustical Society of America 75, 570 (1984); https://doi.org/10.1121/1.390530

The physics of small-amplitude oscillation of the vocal folds

The Journal of the Acoustical Society of America 83, 1536 (1988); https://doi.org/10.1121/1.395910

Aerodynamic transfer of energy to the vocal folds

The Journal of the Acoustical Society of America 118, 1689 (2005); https://doi.org/10.1121/1.2000787

Electroglottographic wavegrams: A technique for visualizing vocal fold dynamics noninvasively

The Journal of the Acoustical Society of America 128, 3070 (2010); https://doi.org/10.1121/1.3493423

On the use of the derivative of electroglottographic signals for characterization of nonpathological phonation

The Journal of the Acoustical Society of America 115, 1321 (2004); https://doi.org/10.1121/1.1646401

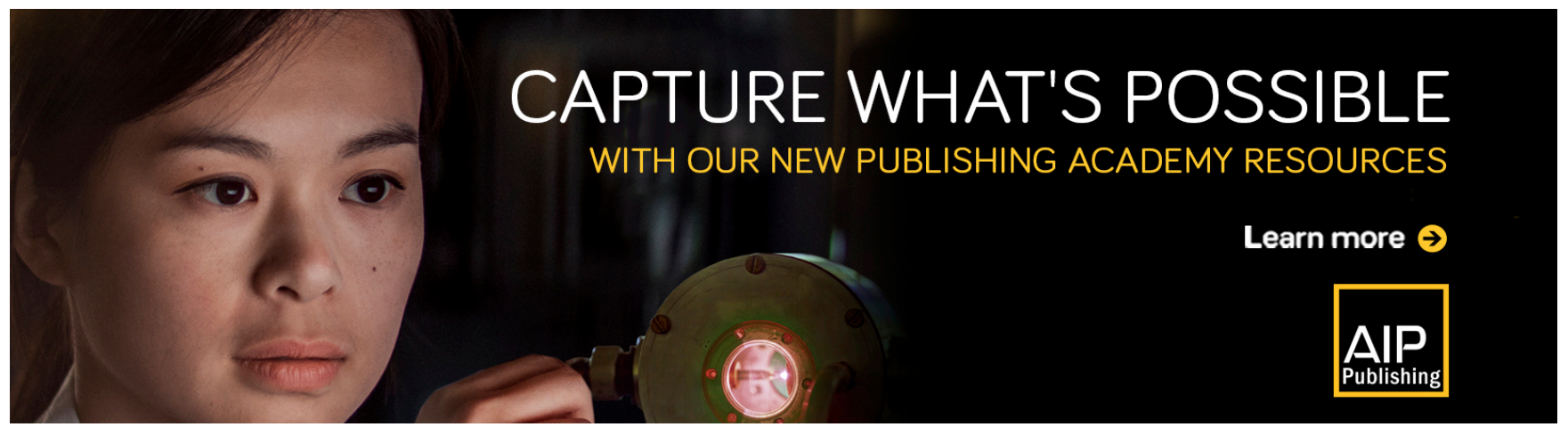




\title{
A model for vocal fold vibratory motion, contact area, and the electroglottogram
}

\author{
D. G. Childers \\ Department of Electrical Engineering, University of Florida, Gainesville, Florida 32611 \\ D. M. Hicks and G. P. Moore \\ Department of Speech, University of Florida, Gainesville, Florida 32611 \\ Y. A. Alsaka \\ Department of Electrical Engineering and Communication Sciences, University of Central Florida, \\ Orlando, Florida 32861
}

(Received 25 August 1985; accepted for publication 1 August 1986)

\begin{abstract}
The electroglottogram (EGG) has been conjectured to be related to the area of contact between the vocal folds. This hypothesis has been substantiated only partially via direct and indirect observations. In this paper, a simple model of vocal fold vibratory motion is used to estimate the vocal fold contact area as a function of time. This model employs a limited number of vocal fold vibratory features extracted from ultra high-speed laryngeal films. These characteristics include the opening and closing vocal fold angles and the lag (phase difference) between the upper and lower vocal fold margins. The electroglottogram is simulated using the contact area, and the EGG waveforms are compared to measured EGGs for normal male voices producing both modal and pulse register tones. The model also predicts EGG waveforms for vocal fold vibration associated with a nodule or polyp.
\end{abstract}

PACS numbers: 43.70.Aj, 43.70.Bk, 43.70.Dn, 43.70.Jt

\section{INTRODUCTION}

For some years we have been assessing electroglottography as a method for investigating the functioning of the vocal folds. For electroglottography to be useful in the speech clinic or laboratory, the electroglottogram (electroglottographic waveform) should be related to vocal fold vibration. Furthermore, any model of vibration should correspond to the reality of laryngeal anatomy. Initial consideration was given to the traditional three-segment conceptualization of the vocal folds (anterior and middle thirds are membraneous; posterior third is cartilaginous). Based on observations from laryngeal films during sustained phonation, the posterior one-third segment was observed to be static, while the anterior two segments were critically involved in the vibratory motion. Therefore, we only needed to model the membraneous portion of the folds. Some of our film observations allowed us to simplify the physiology as though there were an anatomical and physiological symmetry between the anterior and middle thirds of the folds. In other words, these two segments represent mirror images across a coronal section of the larynx through their point of junction. Our model, when based on this assumption, was capable of generating EGG waveforms that were nearly identical to the measured waveforms. This confirmed our assumptions and accounts for the stylized graphic representation of the vocal folds incorporated in our figures. This representation depicts an idealized view of only the anterior one-third segment of the vocal folds. It is our contention that we can take this artistic license due to the mirror image concept mentioned above. We have revised the elementary descriptive EGG model suggested by Rothenberg (1981) until we now believe that the segments of the EGG are related to vocal fold vibratory events as depicted in Fig. 1. These conclusions are drawn from our observations of ultra high-speed laryngeal films synchronized with EGG and speech waveforms (Childers et al., 1982; Childers et al., 1983; Childers et al., 1984; Childers and Krishnamurthy, 1985). The segments of the EGG waveform are most easily described by starting with point 5 in Fig. 1. At this point the glottis has just closed, i.e., the glottal area has just become zero. For a normal male voice in modal register this typically occurs as the lower margins of the vocal folds initially come into contact. Furthermore, we have restricted our data to those cases in which contact of the folds initiates anteriorly and progresses posteriorly in a "zipperlike" manner as shown by the artistic rendition of the vocal folds at the bottom of Fig. 1 . We conjecture that: (1) the contact area of the vocal folds continues to increase from point 5 to 6 to 1 as the folds roll into contact inferiorly to superiorly; (2) the maximum vocal fold contact area occurs at point 1 and is maintained until point 2; and (3) following point 2 the lower vocal fold margins begin to separate posteriorly to anteriorly in a zipperlike manner. The anterior-posterior angle for the separation of the vocal folds differs from the angle assumed for closure. At point 3, the lower margins of the folds are separated and the upper margins are moving apart but lag behind the lower margins. The vocal folds are completely separated at point 4 , i.e., the glottis has just opened. The folds continue to move laterally in the interval following point 4 until maximum glottal opening is achieved. The folds then begin to move medially with glottal closure occurring at point 5 and the cycle repeats. The plausibility of this description will be substantiated in this paper using our complete model of vocal 


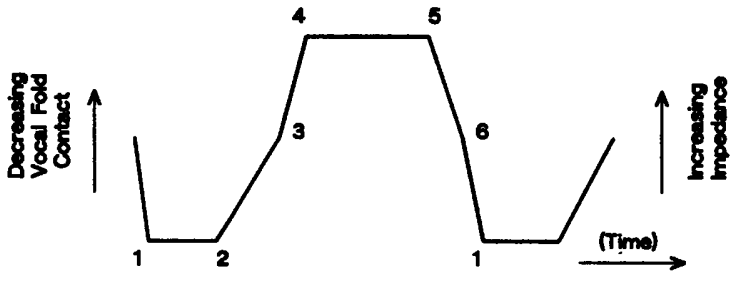

1 - 2 Vocal folds maximally closed. Maximum contact area

2 - 3 Folds parting, usually from lower margine toward upper mergins and posterior to anterior.

3 When this break point is present, this usually corresponds to folds opening along upper margin.

3 - 4 Upper fold margins continus to open.

4 - 5 Folds apart, minimum contact area.

3 - 5 Open phase.

5 Folds in contact along lower margin. Clottal area zero.

5 -6 Folds closing from lower to upper margin and from anterior to posterior.

6 - 1 Rapid increase in vocal fold contact

5 - 2 Closed phase.

ARTISTIC RENDITION OF VOCAL FOLD MOTION

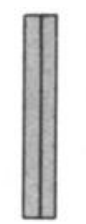

$1-2$

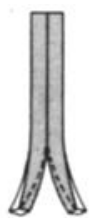

$2-3$

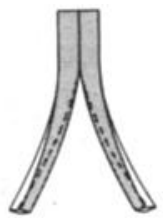

$3-4$

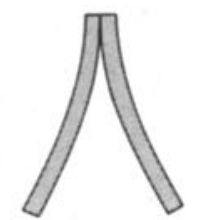

$4-5$
FIG. 1. Idealized descriptive EGG waveform. The vocal fold events are labeled on the EGG waveform and correspondingly on the artistic rendition of the vocal fold motion. The vocal folds are stylized and depict only the anterior one-third segment of the folds. The upper and lower vocal fold margins are out of phase.

fold vibratory motion, contact area, and EGG waveform generation.

The functioning of the electroglottograph as an instrument has been described in Childers and Krishnamurthy (1985). The instrument measures the electrical impedance variations between a pair of electrodes held in contact with the skin on either side of the thyroid cartilage. The stated objective of the device is to register the contact between the vocal folds as the time varying signal we call the EGG. Fourcin (1981) suggests the signal be referred to as the laryngogram because he maintains that the device does not measure glottal area but rather contact area. In any case, the amplitude variations of this signal are generally thought to be representative of the amount of contact between the vocal folds (Fourcin, 1981; Childers et al., 1983; Childers and Krishnamurthy, 1985; Baer et al., 1983a,b; Titze and Talkin, 1981; Gilbert et al., 1984).

The EGG instrument measures tissue impedance. The radio-frequency probe current detected by the EGG device passes from one electrode, the transmitting source, through the laryngeal tissue to the receiving electrode. The tissue impedance varies with the length and composition of the tissue path traversed by the current as well as the number of current paths (tissue cross-sectional area) between the two electrodes. The total impedance is a function of the tissue path length as well as the tissue cross-sectional area and composition. The impedance increases as the folds separate because the current paths become fewer and less direct, hav- ing to pass around the anterior and posterior regions of the larynx. The tissue impedance is a complicated problem and is not fully understood. We generally believe the impedance is least for complete fold contact because in this condition there are, in effect, many parallel conductive paths between the electrodes. The combined total parallel impedance is less than the impedance of any one path. A consequence is that the tissue impedance is inversely proportional to the contact area of the vocal folds. (Recall that the resistance of a substance is inversely proportional to the cross-sectional area of the substance.)

Titze and Talkin (1981) have pointed out that, "The electrical energy transmitted through the larynx (by the EGG device) cannot be focused into a narrow beam. The EGG signal, therefore, cannot be used to probe the spatial nonuniformity of the contact area, but rather, results in an integrated measure of contact over the entire medial surface. Furthermore, a shunt conductance around the glottis is always present." This shunt impedance is at least partially due to gross anatomical laryngeal structures that may move during voicing or voicing onset or offset. These laryngeal movements undoubtedly contribute in part to shunt impedance changes that are reflected as EGG baseline shifts.

These issues concerning the EGG, vocal fold vibratory motion, and contact area are difficult to validate using conventional measurement techniques. Even ultra high-speed laryngeal photography is of little help. Consequently, we decided to model vocal fold vibrations and the EGG waveform. In this paper we describe our model and show how the model predicts EGG waveforms measured from subjects with normal larynges and patients with vocal fold disorders.

\section{RELATIONSHIP BETWEEN MECHANICAL MODELS AND VOCAL FOLD CONTACT AREA}

Much of the research on modeling vocal fold motion may be described as biomechanical (Ishizaka and Flanagan, 1972; Titze and Talkin, 1981; Titze et al., 1983). Titze (1984) described a configurational model of the glottis and vocal folds, which depicted the vocal folds in three dimensions. Various parameters were defined and the prephonatory and time varying vocal fold displacements were described by sinusoidal-like equations. The paper showed that basic features of the glottal area, vocal fold contact area, and glottal flow (volume velocity) can be derived from the combination of prephonatory and dynamic displacement features. Titze also calculated a synthesized EGG waveform (as well as other waveforms) using his programmed equations, which make use of such parameters as abduction quotient, shape quotient, etc. One of Titze's goals was to infer the glottal configuration and aspects of vocal fold movement from analyses of measured glottographic waveforms.

Our goals are substantially those of Titze's (1984). However, we have chosen to translate our observations of vocal fold motion, as observed from ultra high-speed laryngeal films, into a simple three-dimensional geometric model of vocal fold vibrations. To explain how we accomplished this task, recall the simple two-mass vocal fold articulatory speech synthesis model of Ishizaka and Flanagan (1972), 


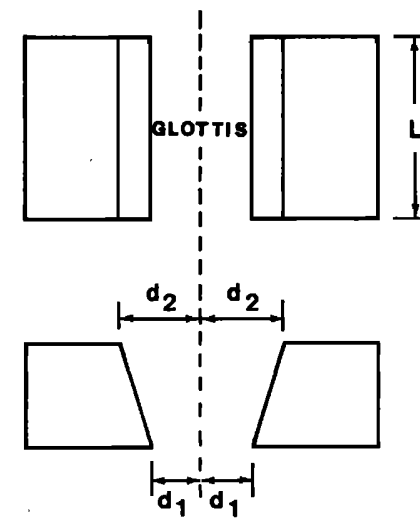

(a)
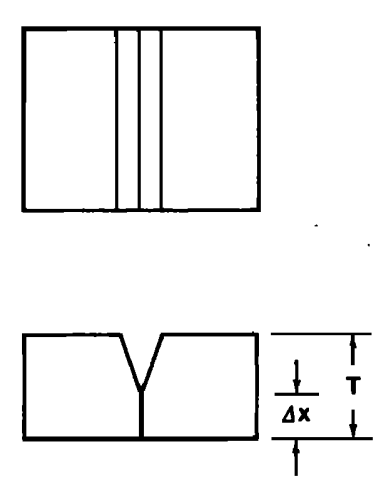

(b)
FIG. 2. Simple elastic one-mass vocal fold model. Superior (upper drawing) and frontal (lower drawing) views. Vocal folds (a) open and (b) partially closed.

Flanagan and Ishizaka $(1975,1976,1978)$, and Flanagan et al. (1980). The Flanagan and Ishizaka two-mass model provides sufficient details to calculate the speech waveform, the pressure and air volume-velocity distributions in the vocal cavities, the motion of the vocal folds, the glottal area, the vibration of the cavity walls, and other factors. A limitation of this two-mass model is that the projected glottal area is always rectangular. The folds are either in contact or separated; there is no gradation of closure or opening. The progression of contact area is stair-stepped; first it is a minimum (open glottis), next there is complete closure, partial contact (lower masses in contact), and finally there is full contact (lower and upper masses both in contact). This approximation does not adequately model the vibratory motion of true vocal folds during phonation.

A more realistic simulation of the vocal folds is achieved with an elastic one-mass model for each fold as depicted in Fig. 2. In the present context, elasticity is defined as a property of matter whereby the mass resists deformation, but will return to its initial form following deformation. The horizontal displacements of the superior $(d 2)$ and inferior $(d 1)$ margins of the simulated vocal folds are given as inputs to the model. These displacements may be determined from observations or from a model of vocal fold movement, e.g., the two-mass model. The wedge is constructed by using a straight line interpolation between points $d 1$ and $d 2$. The thickness of the simulated folds is $T$ and their length is $L$. The ratio of $L$ to $T$ can be specified. In the examples to follow we have set $L / T=5$, a dimensionless ratio. The model assumes an elastic collision between the right and left vocal folds. In Fig. 2 and throughout this paper, we have restricted the deformation of the vocal folds during contact to one dimension, namely, laterally. The effects of this restriction are discussed below. The length of vertical contact $\Delta x$ can then be easily computed and the contact area may be estimated at a particular time instant as

$$
A=(L)(\Delta x) \text {. }
$$

Here, we have only outlined the calculations necessary to determine the contact area. In Sec. II we discuss these calcu-

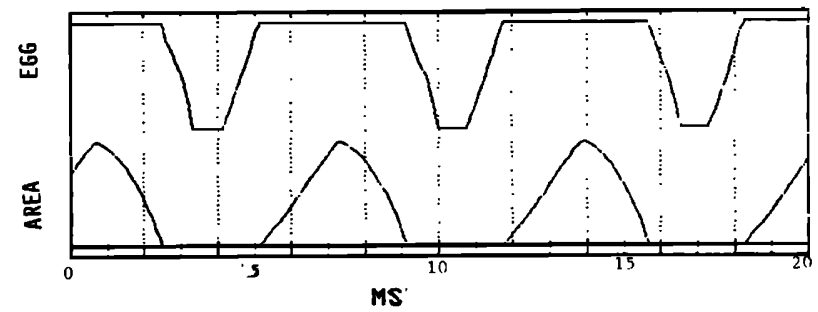

(a)

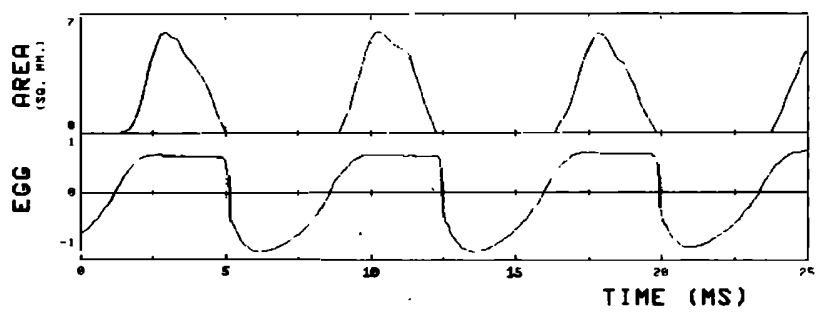

(b)

FIG. 3. (a) EGG and glottal area calculated from the simple elastic onemass vocal fold model. (b) Measured glottal area and EGG waveforms aligned and synchronized in time.

lations in more detail using a more sophisticated vocal fold model. The EGG waveform is related to $A$, which is discussed in detail below. Figure 3(a) shows the EGG signal and the calculated glottal area, both estimated with the aid of this model. Compare these results with Fig. 3(b), which shows a measured EGG and glottal area. This comparison highlights how well our simple model works. Our basic model of the EGG signal is a good first approximation relating glottal opening and glottal closure to EGG events as depicted earlier in Fig. 1. But it does not account for the angle at which the folds move together from anterior to posterior as the glottis closes, or the angle at which the folds open from posterior to anterior as the glottis opens. Note, also, that this model is a simplified method for calculating the area of contact of the vocal folds. Recall that we restricted vocal fold deformation during contact to the lateral dimension only. This is an idealization and does not allow an increase in contact area due to the tissue spread in all directions over the sagittal contact plane. Another limitation of this model is that it does not reflect anatomic reality by not anchoring the vocal folds at the anterior commissure. Consequently, we were led to consider an improved version of this model that accommodates this latter restriction.

\section{VIBRATORY MODEL OF THE VOCAL FOLDS}

Ultra high-speed films of the vocal folds of males in modal register show that a phase difference (i.e., time lag) may exist along the length of the vocal folds during their closing and opening stages. During closure, contact between the folds first occurs over a small portion of their length, and continues, zipperlike, along the length of the folds. This concept is graphically illustrated in Fig. 4, which shows the modeled vocal folds now hinged at the anterior commissure. As the folds move into contact during closure, one can ima- 


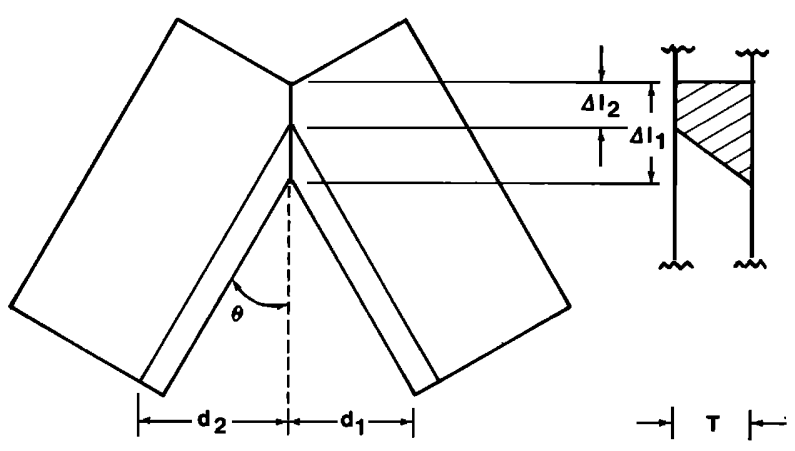

FIG. 4. Hinged elastic one-mass vocal fold model, superior and midsagittal views. This figure can be used for glottal opening and closure and for upper and lower margin displacements.

gine a zipper being pulled from anterior to posterior. The reverse occurs during the opening stage. A second concept that we have incorporated in this model involves the angles at which the glottal area appears to increase and decrease in ultra high-speed larygeal films. This concept is also shown in Fig. 4, labeled as the angle $\theta$, which defines the relationship between the medial edge of each fold and a midsagittal reference line through the glottis. As can be visualized, this angle could be defined differently for both the opening and closing stages during vibration. Variations in the angle parameter will be shown to have a differential impact on segments of the EGG.

A third and final concept included in our model is the vertical (superior-inferior) phase difference between the upper and lower margins of the vocal folds which is often readily observable in our laryngeal films of subjects in modal register. Specifically, the lower margin leads the upper margin in both the opening and closing stages of vibration.

All three of the above concepts are illustrated by the artistic rendition of vocal fold motion in Fig. 1. For computational purposes, however, the illustration in Fig. 4 is the basis for our vibratory vocal fold model. We now relate this model to the calculation of contact area and finally to the prediction of the electroglottogram.

To calculate the vocal fold contact area the model works as follows. The upper and lower vocal fold displacements must be specified or modeled. In this paper, we use the Flanagan-Ishizaka two-mass vocal fold articulatory synthesis model to first calculate the upper and lower glottal areas, denoted as AG2 and AG1, respectively. Please note that we restrict our attention to only these two parameters of the numerous parameters used in the Flanagan-Ishizaka model. In our model, the displacements $d 2$ and $d 1$ of the opposing vocal fold margins (both upper and lower) from the midsagittal line are calculated for a particular time instant $n$ as

$$
\begin{aligned}
& d 1(n)=\operatorname{AG} 1(n) / 2 L, \\
& d 2(n)=\operatorname{AG} 2(n) / 2 L,
\end{aligned}
$$

where $n$ is a time index and $L$ is the length of the vocal folds. These displacement values are used to position the upper and lower margins at the posterior ends of the vocal folds in the model in Fig. 4. This model has a triangular glottal area with an angle $\theta$ as shown.

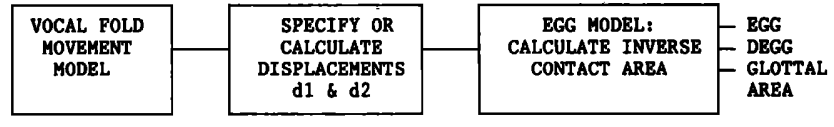

FIG. 5. EGG waveform simulation using a vocal fold movement model, e.g., the Flanagan-Ishizaka two-mass vocal fold articulatory speech synthesis model.

The vocal fold contact area is calculated using the threedimensional configuration of Fig. 4. Several conditions may be specified in the computer program implementation of this model: (1) The folds are not in contact (no contact area); (2) the lower margins of the folds are in contact and the upper margins of the folds are not in contact (area of contact is triangular in form); (3) lower margins are not in contact and the upper margins are in contact (area of contact is again triangular); and (4) both upper and lower vocal fold margins are in contact and possibly out of phase (area of contact is trapezoidal in form, as shown in Fig. 4). With these conditions, the EGG waveform is defined as

$$
\operatorname{EGG}(n)=k /[A(n)+C],
$$

where $n$ is the time index, $A(n)$ is the contact area, $C$ is a constant proportional to the shunt impedance specified for the case when $A(n)=0$, and $k$ is a scaling constant. The glottal area is calculated in the model using the projected triangular glottal area configuration, not the projected area given by AG1 and AG2 of the Flanagan-Ishizaka two-mass vocal fold articulatory speech synthesis model. The EGG model described above is summarized in Figs. 5 and 6.

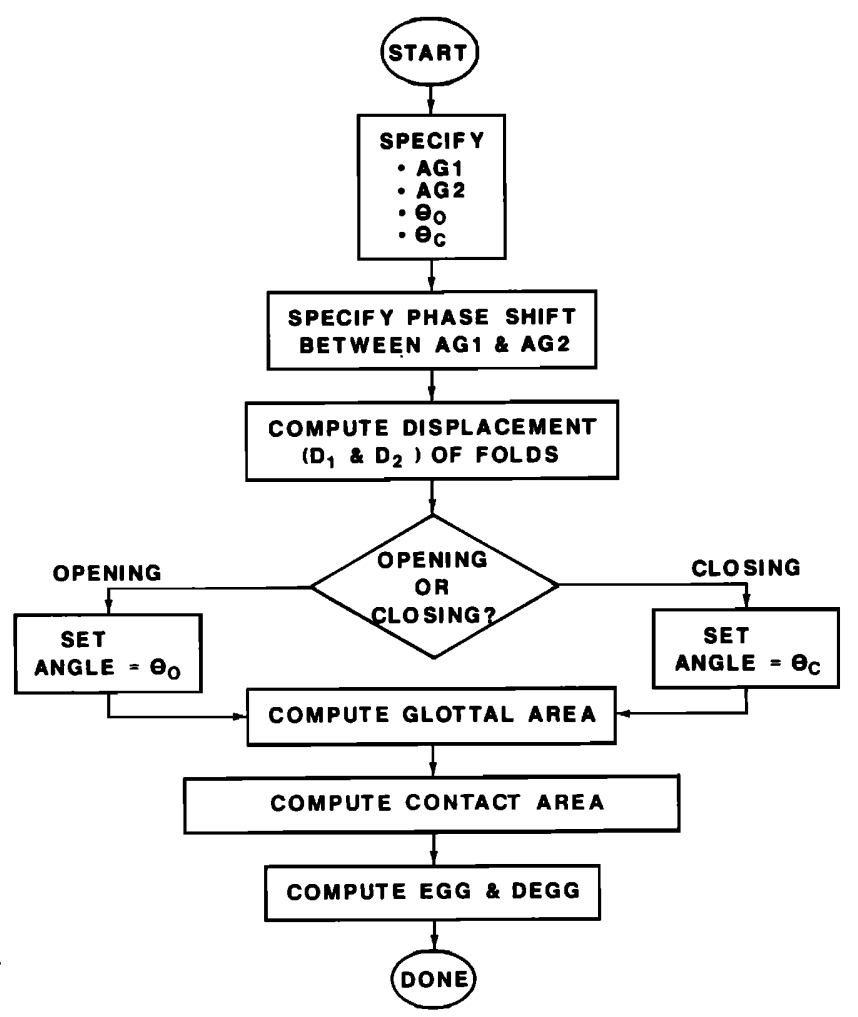

FIG. 6. Flow chart for EGG model calculations when using the FlanaganIshizaka model. 


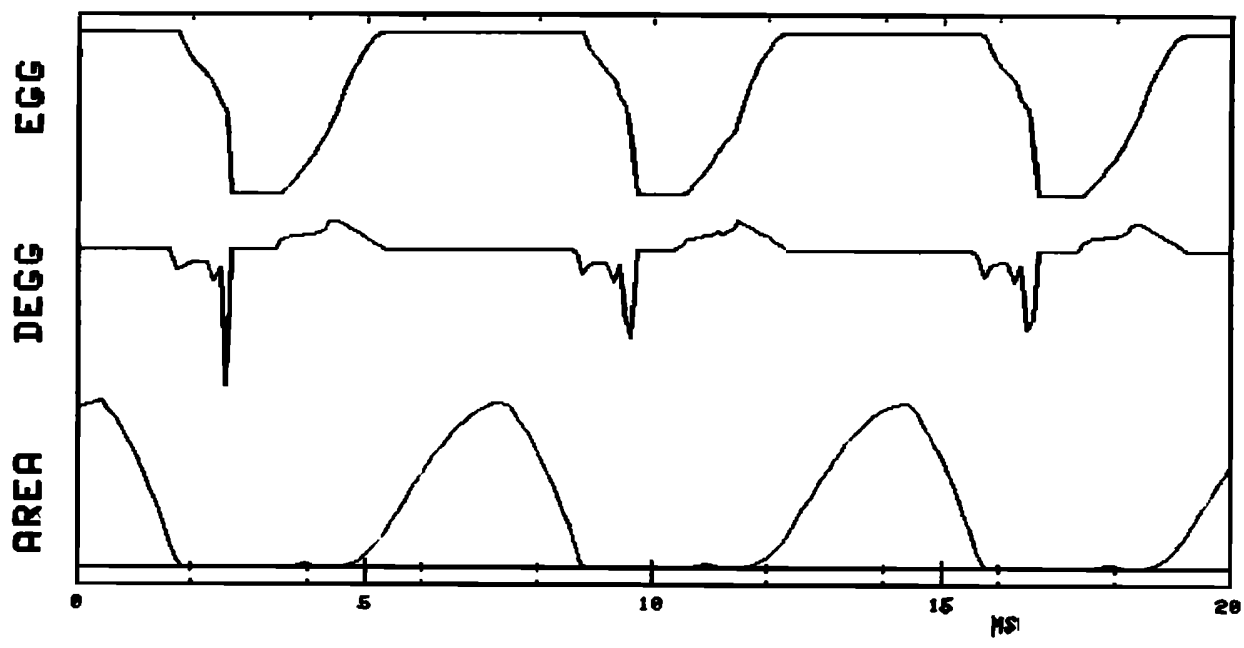

FIG. 7. Simulated EGG, DEGG, and glottal area waveforms with $\theta_{o}=1.0^{\circ}$, $\theta_{c}=0.2^{\circ}$, and a $0.7-\mathrm{ms}$ time lag between upper and lower vocal fold margins.

\section{SIMULATION RESULTS}

In the examples to follow, the three vocal fold vibratory concepts discussed previously are the basis of experimental manipulation in our model. To effect simulation, these concepts are translated into parameters. The first concept, the zipper effect, is foundational to the other two, as it represents one common mode of vocal fold vibration. The second concept establishes the angles of closure $\theta_{c}$ and opening $\theta_{o}$. The third and final concept relates to the phase difference between the upper and lower margins of the folds, which is captured by a "lag time" parameter.

To help orient the reader, the first example is illustrated in Fig. 7 for the following conditions: opening angle $\theta_{o}=1.0^{\circ}$, closing angle $\theta_{c}=0.2^{\circ}$, and a lag of $0.7 \mathrm{~ms}$ between the upper and lower vocal fold margins. These values have been found to simulate features of an actual EGG quite well. Subsequent examples demonstrate the effects on the EGG waveform of varying one parameter at a time. The lag can be specified in the model as a fraction of the fundamental period of voicing. Here, we represent this lag in units of ms. The maximum and minimum displacements of the upper and lower margins are the same. There is a strong resemblance between this example's simulated EGG waveform and the measured EGG waveform in Fig. 3(b), except the simulated waveform does not have the rounded corners of the measured waveform. The differentiated EGG (DEGG) wave shown in Fig. 7 is calculated as the derivative of the EGG waveform. The DEGG has been assumed to be useful for identifying instants of glottal closure and opening (Childers and Krishnamurthy, 1985). But even this simple example illustrates the tenuousness of this hypothesis. For example, the instant at which the largest negative peak occurs in the DEGG has been considered to be a good approximation for the moment of glottal closure. Figure 7 shows this assumption to be incorrect. The first small negative peak in the DEGG, which corresponds to the first knee in the downward going segment of the EGG, is a more accurate prediction of glottal closure than the large negative peak. Similarly, the location of the largest positive peak in the DEGG has been suggested as an estimate of the instant at which the glottis opens. This estimate is approximately cor- rect for this example, but often this peak is quite broad, making the decision of the exact instant of glottal opening uncertain.

We now use our complete model of vocal fold vibration and EGG waveform generation to show the effects on the EGG of (1) varying the opening angle of the vocal folds, (2) varying the closing angle of the vocal folds, (3) varying the phase difference between the upper and lower margins of the folds, (4) simulating a mucus strand bridging the vocal folds during the glottal opening stage, and (5) simulating vocal fold polyps or nodules.

\section{A. Varying the opening angle}

For these calculations, the closing angle was fixed at $\theta_{c}=0.2^{\circ}$. Figure $8(a)$ shows the effects of varying $\theta_{o}$ from $0.2^{\circ}$ to $2.7^{\circ}$. As $\theta_{o}$ increases, the rising slope of the EGG, which corresponds to the opening phase of the glottal area waveform, decreases. A bend in the rising portion of the EGG is visible. For $\theta_{o} \geqslant 3.5^{\circ}$, the EGG rises gradually as the model of the vocal folds opens until the angle of opening reaches the specified $\theta_{o}$ at which point the EGG jumps suddenly, in steplike fashion, to the maximum EGG value. This discontinuity phenomenon is due to the constant $C$ being specified for small values of $\theta_{o}$ and then left unchanged for all succeeding calculations. This problem might be resolved by letting the constant $C$ be a function of the opening angle. Note that the differentiated EGG (DEGG) waveform marks the approximate instant of glottal opening and closing with its positive and negative peaks, respectively. However, as the angle of opening increases the positive DEGG peak becomes broader, making the decision for the instant of glottal opening more difficult. For our data, a good EGG model waveform is best simulated using $0.5^{\circ} \leqslant \theta_{o} \leqslant 2^{\circ}$.

\section{B. Varying the closing angle}

For these calculations the opening angle was fixed at $\theta_{o}=2^{\circ}$. Figure 8(b) shows the effects of varying $\theta_{c}$ from $0.0001^{\circ}$ to $2^{\circ}$. For $\theta_{c}>5^{\circ}$ a stair-step discontinuity occurs in the falling slope of the EGG, which corresponds to the clos- 


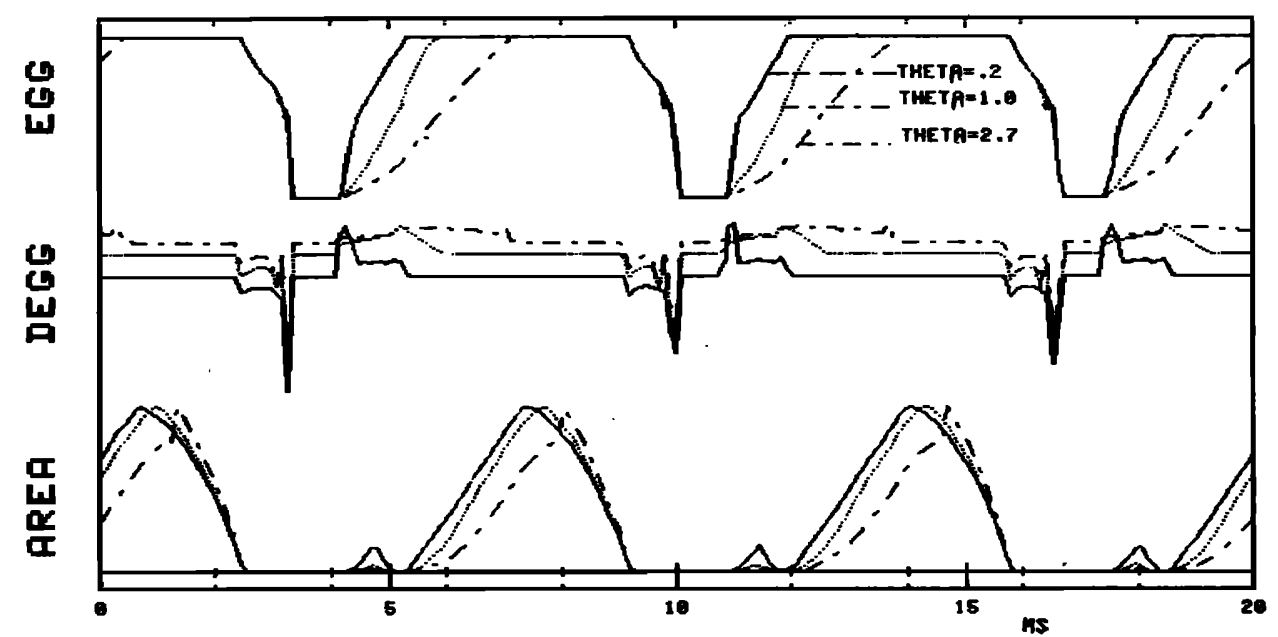

(a)

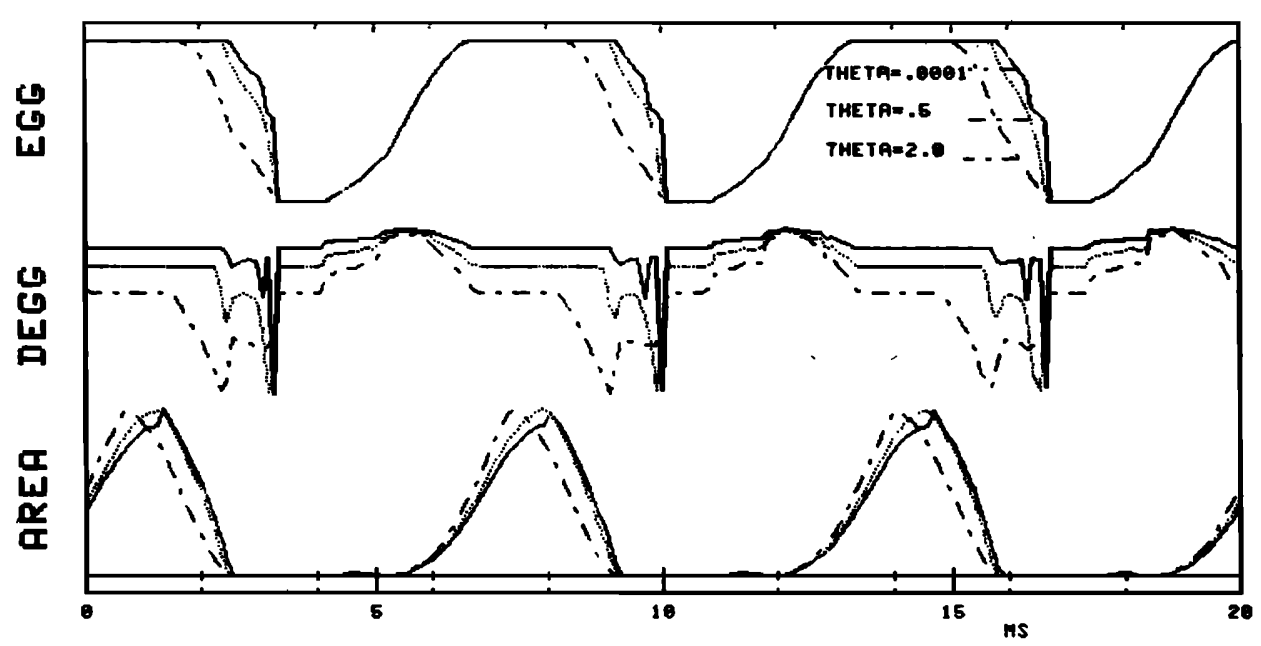

(b)
FIG. 8. Simulated EGG, DEGG, and glottal area waveforms for various angles of glottal (a) opening $\theta_{o}$ and (b) closing $\theta_{c}$. ing phase of the glottal area. As $\theta_{c}$ increases, the magnitude of the falling slope of the EGG decreases.

For small closing angles the largest negative peak in the DEGG waveform does not occur at the instant of zero glottal area, but rather several ms later. However, when the angle of closure is quite large, namely $\theta_{c}=2^{\circ}$, then the large negative peak in the DEGG corresponds approximately to the instant at which the glottal area becomes zero. This appears to agree with actual measured data. The instant of zero glottal area corresponds to the instant at which the EGG starts its downward (negative) deflection. This instant generally occurs in the model waveforms slightly before the instant of greatest negative slope, i.e., the instant at which the DEGG has its largest negative value. These observations relating the DEGG to various sizes of the glottis apply to the model and may not reflect physiological reality. A good EGG model waveform is best simulated using $0.0001^{\circ} \leqslant \theta_{c} \leqslant 0.5^{\circ}$.

\section{Varying the phase difference between upper and lower vocal fold margins}

For these calculations $\theta_{c}=0.2^{\circ}$ and $\theta_{o}=2^{\circ}$, the minimum and maximum upper and lower margin displacements, are equal, and the upper vocal fold margin lags the lower margin. The simulated EGG waveforms in Fig. 9(a) closely resemble the measured EGG for a normal voice phonation at low frequency except for the lack of rounding of the modeled EGG waveform during the interval in which the glottis is closed. When the phase difference (or lag) between the vocal fold margins is zero, the resulting simulated EGG is stylized with a steep closing phase and a gradual opening phase. As the lag is increased in Fig. 9(a), the period of each modeled EGG waveform increases correspondingly. This is an artifact of the present model because all waveforms are obtained by initially starting with the parameters $\operatorname{AG1}(n)$ and AG2 $(n)$ calculated from the Flanagan-Ishizaka model. The zero lag waveform has $\mathrm{AG} 1(n)=\mathrm{AG} 2(n)$ with no lag between AG1 and AG2. The other waveforms have the designated lags between AG1 $(n)$ and AG2 $(n)$, which, in turn, cause variations in the calculated contact area $A(n)$. In the model, the period of the contact area increases with increasing lag. This "artifact" can be manually corrected in the calculations, but we chose not to do so. A revised version of the model will make this correction automatically.

For lag values varying from 0.3 to $0.7 \mathrm{~ms}$, the falling slope of the EGG is affected in a manner analogous to the 


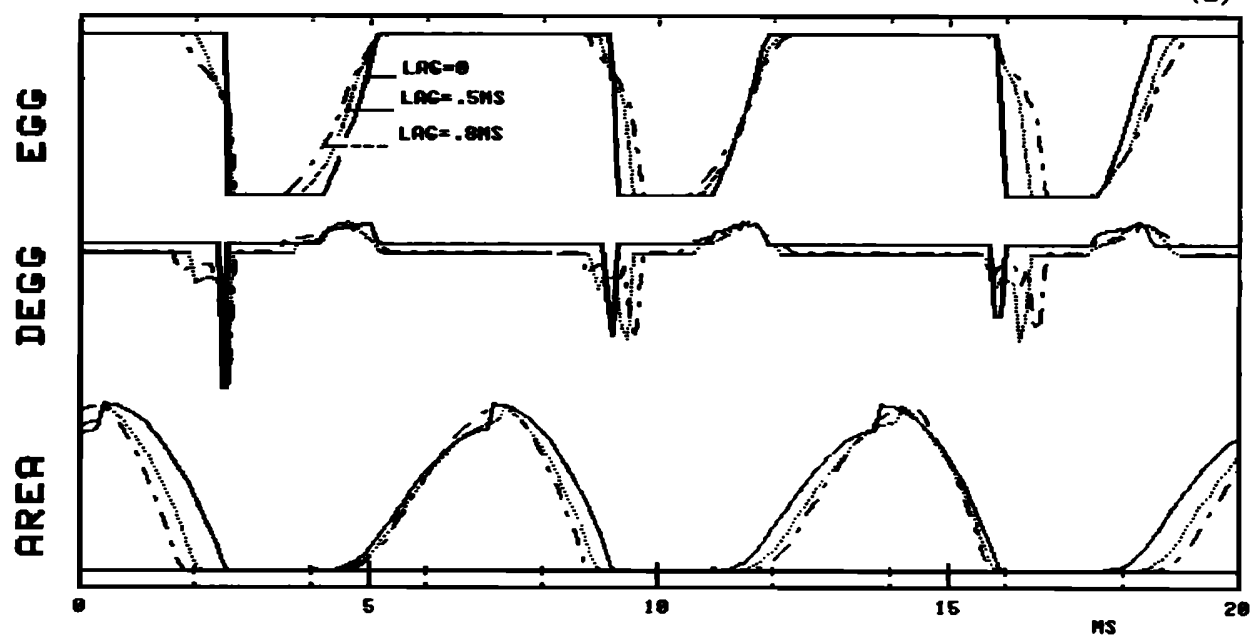

(b)

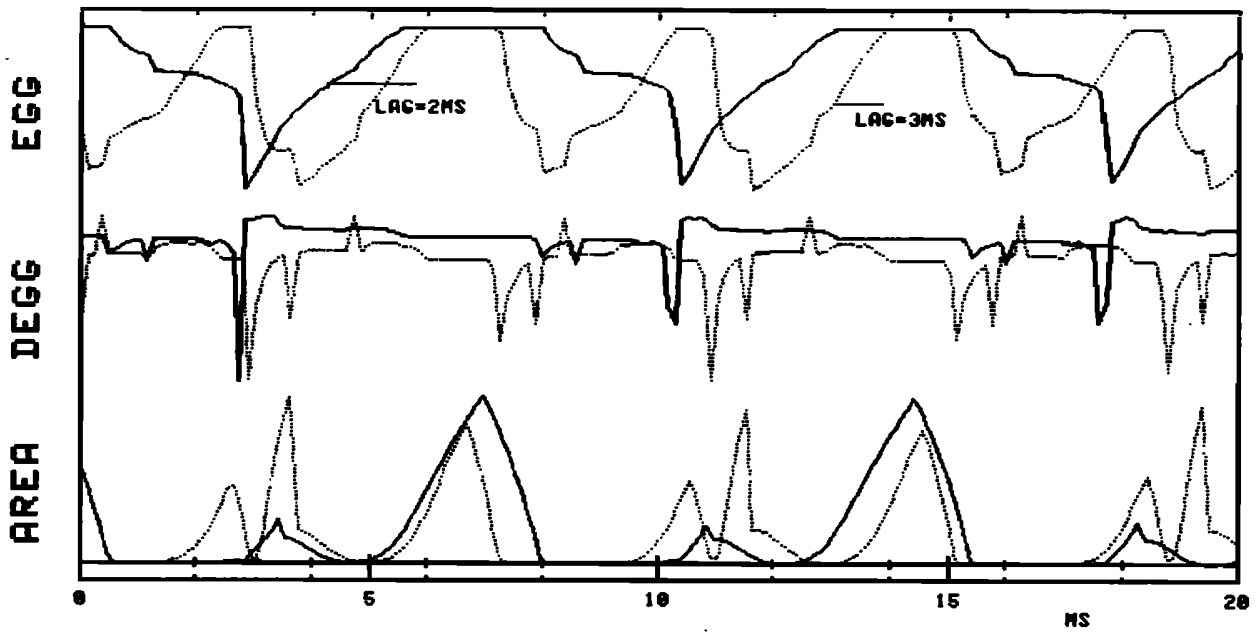

FIG. 9. Simulated EGG, DEGG, and glottal area waveforms for (a) various lag (phase) differences between upper and lower vocal fold margins. The upper margin lags the lower margin for (b) simulated vocal fry and (c) measured vocal fry. Compare the measured EGG (c) with the simulated 2-ms lag EGG in (b).

(c)

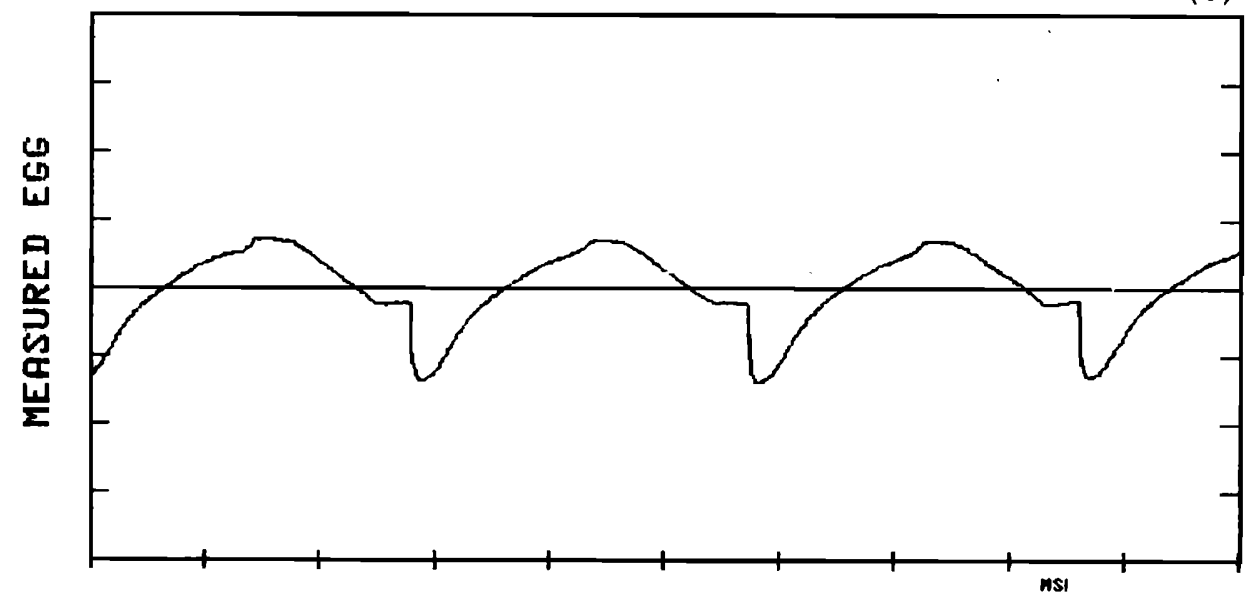

case when the closing angle is increased. When the lag varies from 0.7 to $1.0 \mathrm{~ms}$, discontinuities (steps) occur in both the rising and falling slopes of the EGG, as discussed earlier. With the lag varying from 1.3 to $4.2 \mathrm{~ms}$, the simulated EGG resembles the waveform produced by an individual phonating in vocal fry. [See Fig. 9(b) and (c).] Even for relatively small lags, note that the DEGG waveform does not correctly mark the instants of glottal opening and closure as has been commonly assumed. This phenomenon occurs, e.g., during closure, because the glottal area becomes zero prior to the time at which maximum contact area occurs. This may happen because the folds move into contact along the lower margin, closing off the glottis, prior to making maximal contact along the medial surface. This can also happen when vocal 
fold polyps and nodules are present. Consequently, another criterion is needed for marking the instants of glottal opening and closure when "irregular" or "unusual" vocal fold vibrations occur.

\section{Effects of mucus}

If mucus strands bridge the vocal folds during the opening stage, they may have subtle effects on the EGG waveform. During the opening stage, the EGG rises relatively slowly as the mucus strand stretches but continues to bridge the glottis. At some point in the opening stage the mucus strand breaks. This event can be measured from ultra highspeed laryngeal films. Just after the break, there can be a rapid rise (almost an upward step) in the EGG waveform. This phenomenon is seen even more easily when measured from graphs of the glottal length. For example, as the folds separate, the length of the glottis increases from posterior to anterior (for the data we have restricted ourselves to in this paper). Length is defined as the continuous anterior to posterior distance through the middle of the glottis. If a mucus strand is present, then the effective length of the glottis is less than it would be with the mucus bridge broken. Air does not flow through that portion of the glottis where the mucus strand bridges the glottis, i.e., the air flow is routed around the mucus strand. The effect of the mucus strand, with respect to air flow, is the same as though the folds were still unseparated over the width of the strand. When the mucus strand suddenly breaks, the length of the glottis suddenly increases by the width of that strand since the folds beneath the mucus strand have continued to separate. The increase in the length of the glottis under these conditions is often more dramatic than that for the glottal area.

An example of a measured EGG waveform with a large amount of mucus present on the vocal folds and with a mucus strand bridging the glottis during the opening stage is shown in Fig. 10(a). The mucus phenomenon described above is more readily observable but still subtle in the graphs of glottal length and glottal area. These graphs have a "bend" in the rising portion of the waveform. The folds are open at the beginning of the bend, but a strand of mucus bridges the folds. When this strand breaks, the length waveform rises sharply to its characteristic flat top. While the EGG waveform appears normal for this case, the differentiated EGG does not have its maximum at the instant of glottal opening; rather, the maximum occurs just as the mucus strand breaks. This point corresponds to the "knee" in the opening stage of the glottal area waveform. The EGG (a)

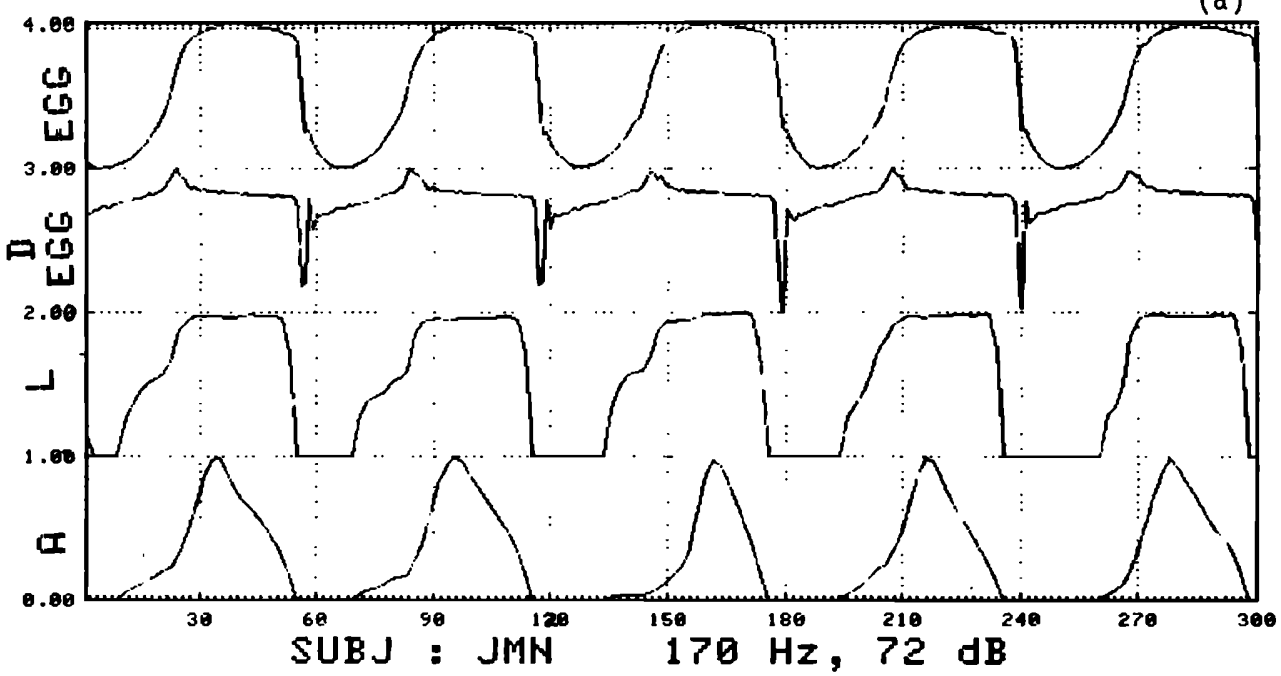

(b)

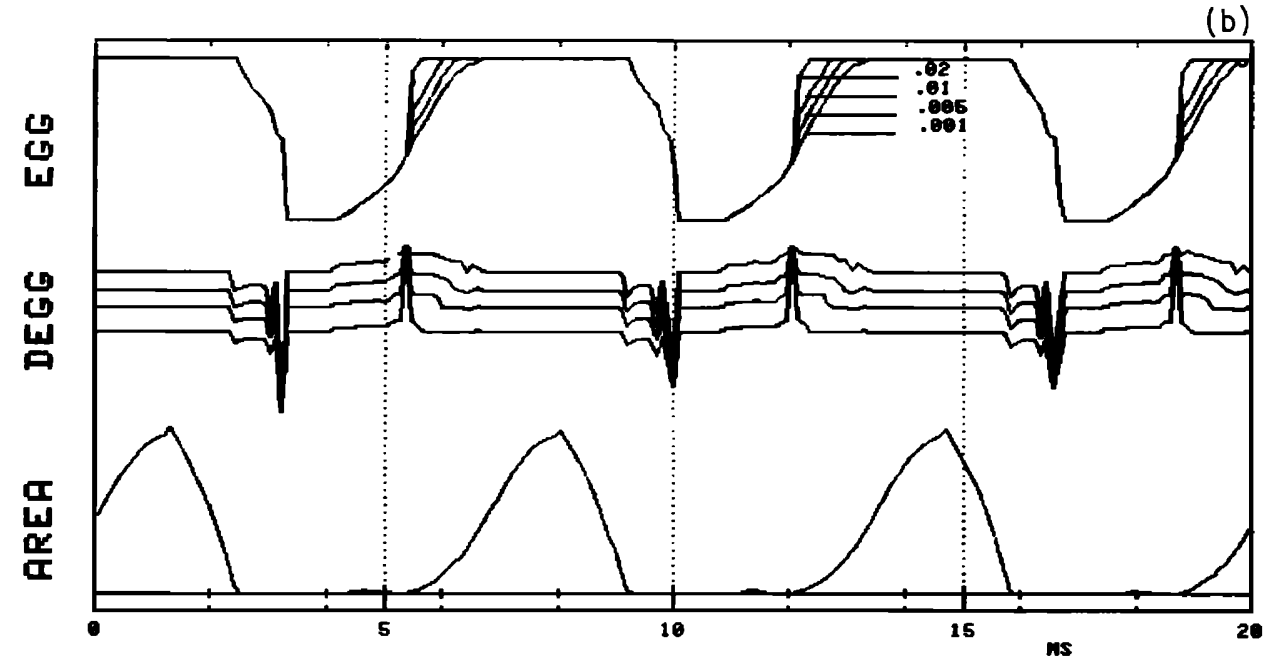

FIG. 10. (a) Measured EGG (upper graph) when mucus strand is present on vocal folds. Also shown are the DEGG, the glottal length $(L)$, and the glottal area $(A)$. (b) Simulated EGG (lower graph) for a simulated mucus strand bridging the vocal folds at $0.15 L$ from the anterior point. The various EGG curves represent a fractional decrease in the vocal fold contact area, which is used in the model to simulate the effect of the mucus strand. The 0.02 curve represents the EGG for a $2 \%$ decrease in contact area. For this case $\theta_{o}=2^{\circ}, \theta_{c}=0.2^{\circ}$. 


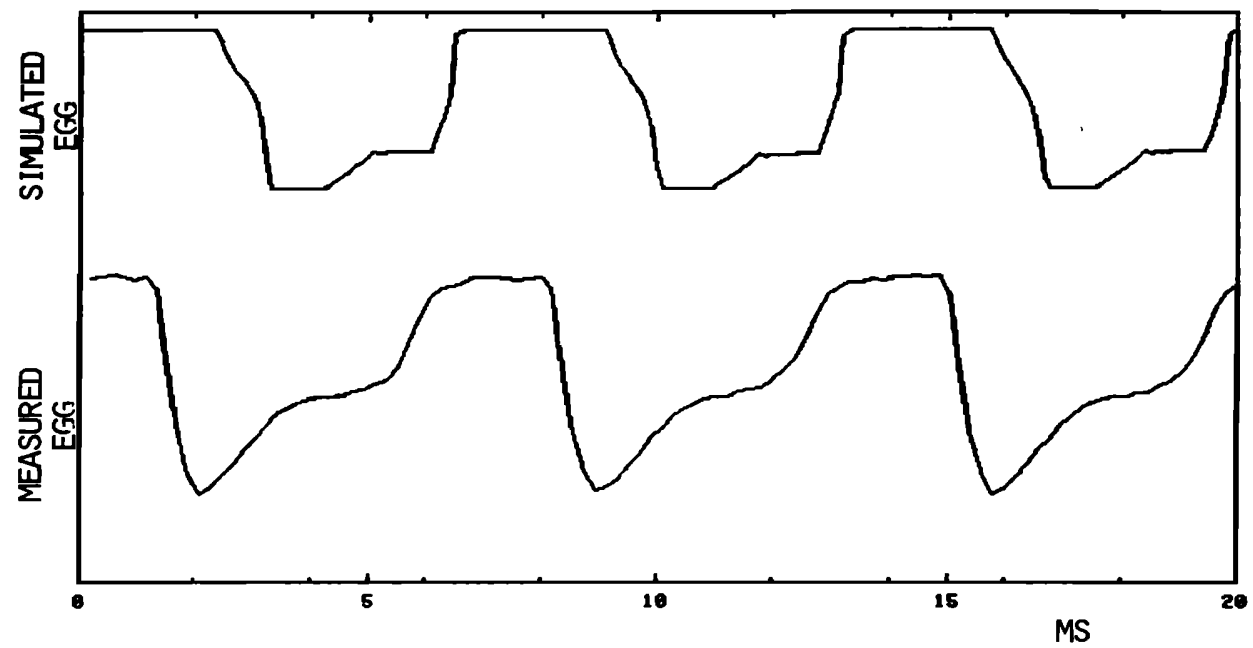

FIG. 11. Simulated EGG (upper graph) for a simulated nodule present on one vocal fold, $\theta_{o}=2.0^{\circ}, \theta_{c}=0.5^{\circ}$. Measured EGG (lower graph) for a nodule present on one vocal fold (time scale adjusted for illustrative purposes).

reaches its maximum just as the mucus strand has broken. Thus an excessive amount of mucus, providing a highly conductive current path, may distort the EGG measurement. In such cases, the EGG may not be an accurate representation of the area of contact of vocal fold tissue. Figure 10(b) shows the effect of simulating various size mucus strands bridging the vocal folds during the initial part of the vocal fold opening stage and then breaking as the folds separate. This simulation mimics the measured event quite well.

\section{E. Effects of nodules and polyps}

Polyps and nodules on the vocal folds arise as a result of trauma to the folds. A laryngeal polyp may result from a single brief period of vocal strain, whereas a nodule usually develops over a longer period of time and progresses through several stages.

The shape, location, and consistency of the polyp or nodule affects the shape of the EGG waveform. We believe that a soft edematous polyp or nodule may have a more pronounced effect on the EGG than a firm or fibrous polyp or nodule.

A large polyp will cause an earlier contact of the vocal folds during the closing stage but not a greater area of contact unless it is very soft. A large protrusion may actually reduce the total amount of contact since there will be no contact adjacent to the nodule. However, since nodules are in the loose mucosa, they slide onto and off the upper surface and consequently may present a different rate and amount of contact between the opening and closing stages of vibration. Furthermore, the EGG for hard protrusions should indicate smaller contact area than might be expected for soft protrusions.

The influence of an edematous polyp on the EGG may be computer simulated in our model by programming a percentage increase (decrease) in the area of contact as the folds move together (or separate). Since the vocal fold model accounts for the geometric configuration and dimension of the folds, we may program, in effect, the location and size of the polyp or nodule by manipulating the duration of area of contact. In principle, we should also be able to program changes in tissue (or mucus) resistivity as well, if and when such values are known.

Figure 11 shows an EGG measured from a patient with a nodule located near the middle of the upper margin of the vocal folds. As the folds begin the glottal opening stage, the

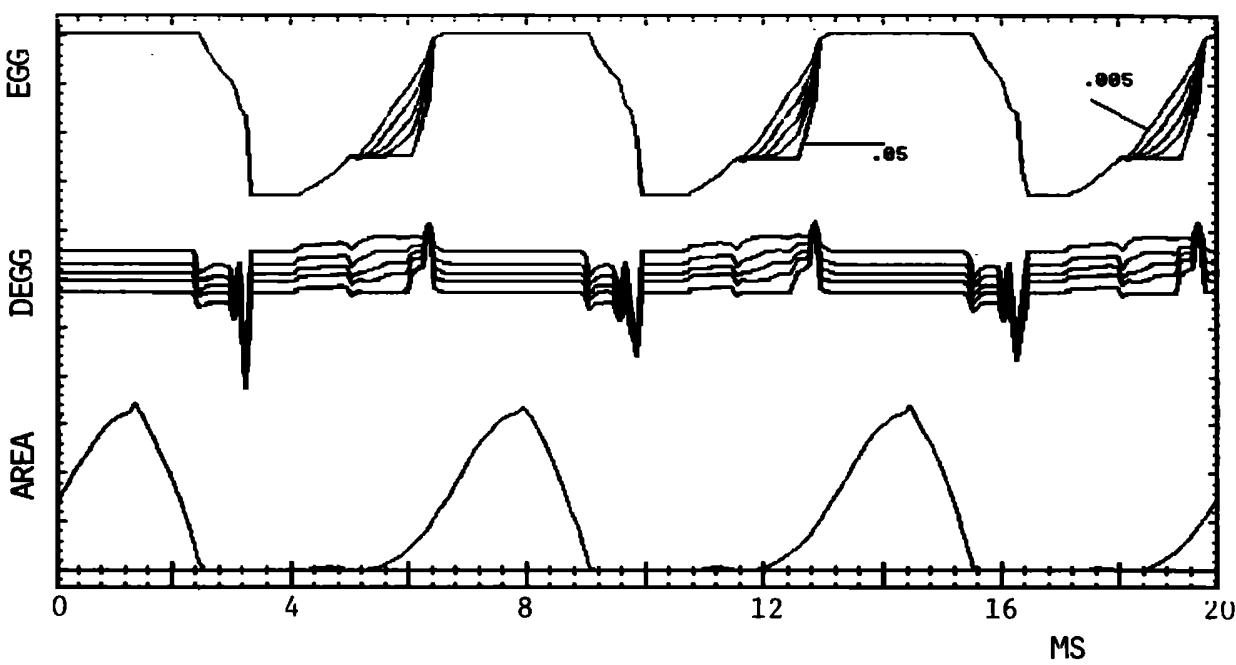

FIG. 12. Simulated EGG waveforms for various fractional increases in contact area, simulating various nodule sizes. The 0.05 curve represents a $5 \%$ increase in the contact area. 


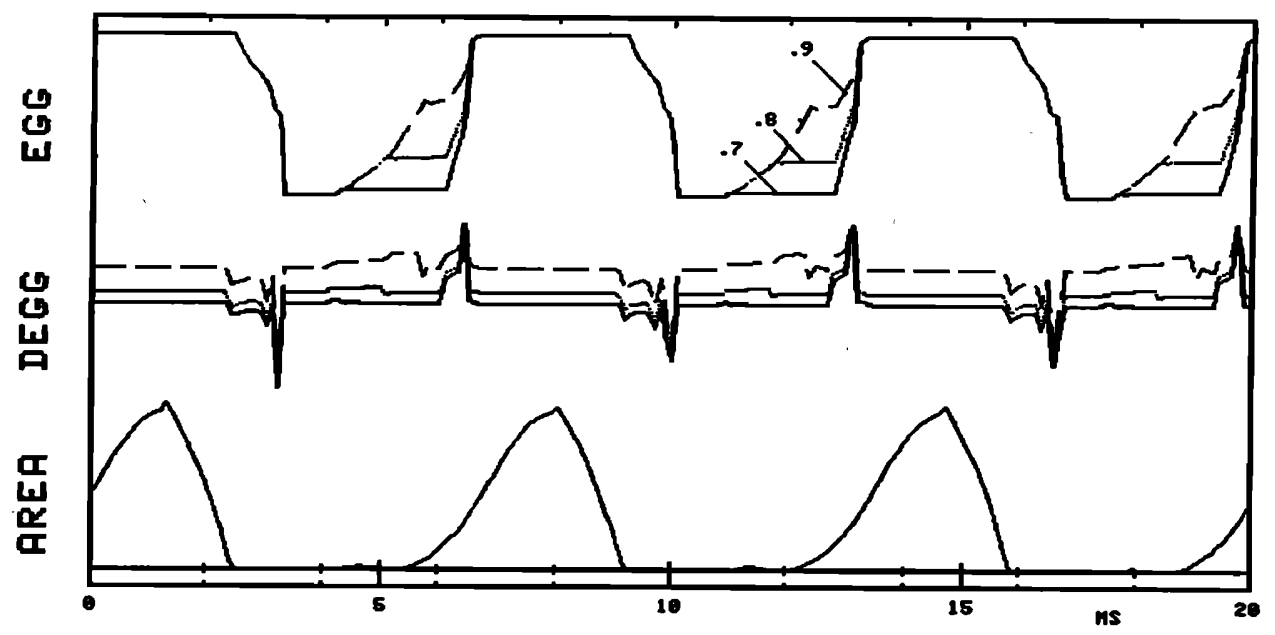

FIG. 13. Simulated EGG when the simulated nodule location is varied posterior-anterior, $\theta_{o}=2.0^{\circ}, \theta_{c}=0.2^{\circ}$. The 0.7 curve denotes that the nodule is located at $0.7 L$ from the posterior boundary of the idealized glottis. The nodule is simulated with a $5 \%$ increase in contact area.

EGG progresses in a normal fashion. But as the folds continue to separate beyond the location of the nodule, the EGG level remains constant. During this interval, the nodule on one fold remains in contact with the other fold for a brief period. The total area of contact remains constant since for this interval the contact area of the nodule compensates for the loss of contact area created by the folds separating below and beyond the nodule location. Consequently, even though the folds are separating around the nodule, the contact area remains essentially unchanged because the previously compressed nodule tissue spreads over the surface of the opposing fold. This same figure shows an EGG waveform for a nodule simulated using the procedures we have just described.

Figure 12 illustrates the effect of varying the area of contact of the nodule on the rising portion of the EGG waveform, corresponding to the separation of the vocal folds. The larger the edematous tissue of the nodule, the longer this tissue can maintain and/or increase the contact area: consequently, the longer the flat segment of the rising portion of the EGG waveform. The location (anterior/posterior) of the nodule can be varied in the model as well, as seen in Fig. 13. If these examples are representative of actual physiology, then they illustrate the potential of the EGG to estimate the size and location (anterior/posterior) of a vocal fold nodule or polyp.

\section{DISCUSSION}

Our simple model of vocal fold vibration and EGG waveform generation does not include all the features of laryngeal function. We stress that it is presently best for a normal male voice in modal register. This is because the vocal folds are apparently thicker for males and our observations concerning opening and closing angles and upper and lower margin lags have been made primarily from ultra high-speed laryngeal films for this case. Higher pitched voices (both males and females) tend to have thinner, longer vocal folds. The initial point of vocal fold contact in this case may occur at the middle of the vocal folds and complete closure may not occur. The folds also appear to be quite thin, with perhaps only one margin, i.e., no upper and lower vocal fold margins. These concepts can be modeled, but we have yet to do so.

Several interesting observations can be drawn from our results. The differentiated EGG (DEGG) has been considered to be a reliable indicator of the instants of glottal opening (large positive peak in the DEGG) and closure (large negative peak in the DEGG). This assumption appears to be valid for a normal male voice in modal register with vocal fold closure. However, the simulation of vocal fold vibratory motion, that either departs from modal register or is impaired by a vocal fold nodule, suggests that the DEGG is no longer a reliable indicator of the instants of glottal opening and closure.

A factor plaguing the users of the EGG is determining the completeness of glottal closure; i.e., can a closed glottis be determined from the EGG waveform alone? Presently, the answer is no! The EGG waveform of a breathy voice with an open glottal chink may look essentially the same as an EGG waveform with complete glottal closure. If the determination of complete glottal closure is important, then the investigator must use other confirming means to assess whether or not complete closure has occurred.

The model is presently inadequate with respect to duplicating the rounded segments of measured EGG waveforms. These rounded segments are most prominent in the closed and open glottal stages. During the closed stage, we feel that real vocal fold tissue may deform to increase (or decrease) the area of vocal fold contact. Our model does not duplicate this phenomenon, but could be modified to do so. During the opening stage, a different phenomenon occurs for which we do not have a ready explanation. Apparently, the tissue conductance path changes gradually as the folds separate. This path probably should not be modeled by a fixed impedance or fixed area (the parameter $C$ in our model). More work is needed to understand this phenomenon better. Despite these limitations of the model, we have replicated many aspects of the EGG waveform via the concept of vocal fold contact area. The numerous examples illustrated in Sec. III substantiate this claim.

The contour of the modeled EGG is sensitive to features of vocal fold vibratory motion as a result of their impact on vocal fold contact area. However, the absolute magnitude of 
the EGG waveform in our model has little importance, since the waveform amplitude can be scaled using the constant $k$ in our model. This is in agreement with our own observations (Childers et al., 1983) and those of Baer et al. (1983a).

\section{CONCLUSIONS}

Our model of the vocal folds has incorporated three concepts of vocal fold vibration. These are: (1) The folds close and open in a zipperlike manner; (2) in a sequence of vibratory cycles, the opening and closing angles of the folds may differ from each other; and (3) a phase lag may exist between the upper and lower vocal fold margin movements. The origin of these concepts comes from observations of ultra high-speed laryngeal films of normal males phonating in modal register. These three concepts led to the creation of a vocal fold vibratory model from which calculations of fold contact area were made. As the folds vibrate, the area varies, affecting the EGG contour. Upon implementation of this complete model we found that simulated EGGs best replicated measured EGGs when

$$
\begin{aligned}
& 0.5^{\circ} \leqslant \theta_{o} \leqslant 2^{\circ}, \\
& 0.0001^{\circ} \leqslant \theta_{c} \leqslant 0.5^{\circ}, \\
& 0.3(\mathrm{~ms}) \leqslant \mathrm{lag} \leqslant 0.7(\mathrm{~ms}) .
\end{aligned}
$$

The model was tested by simulating EGGs for vocal fry register and for selected examples in which nodules or polyps were added to the vocal folds. We also used the model to explain the presence of anomolies in the EGG or DEGG when mucus may bridge the glottis.

One observation about vocal fry is in order. Vocal fry is defined perceptually to be a low-pitched, rough sounding phonation. The model can simulate a frylike EGG waveform at any pitch because the lag between the upper and lower margins may be specified independent of the fundamental frequency. We plan to use our articulatory speech model to synthesize speech at various pitch frequencies and record the listener's report of the auditory perception of such simulations. This will allow us to contrast the vibratory basis of vocal fry with higher-pitched vocal roughness. We are also working on simulating EGG waveforms for high-pitched voices, e.g., female voices, and for other voice registers and voice qualities.

Adjustments in the vocal fold model are also in order. The one-mass vocal fold model depicted in Fig. 1 can be improved by extending the model to include two masses, as shown in Fig. 14. This improved model would more accurately depict the vocal fold vibratory motion by allowing the glottis to close with only partial (approximately one-half, lower masses) vocal fold contact. This addition to the model, including those described in Sec. IV, should markedly improve our ability to relate vocal fold vibratory motion events to EGG waveform segments. Future improvements to our model would include the ability to "draw" a vocal fold configuration, including a nodule, on the screen of a computer terminal. The computer program would then make the required calculations using this configuration. Waveforms of the EGG, glottal area, contact area, DEGG, and others would be calculated and plotted. An additional improve-
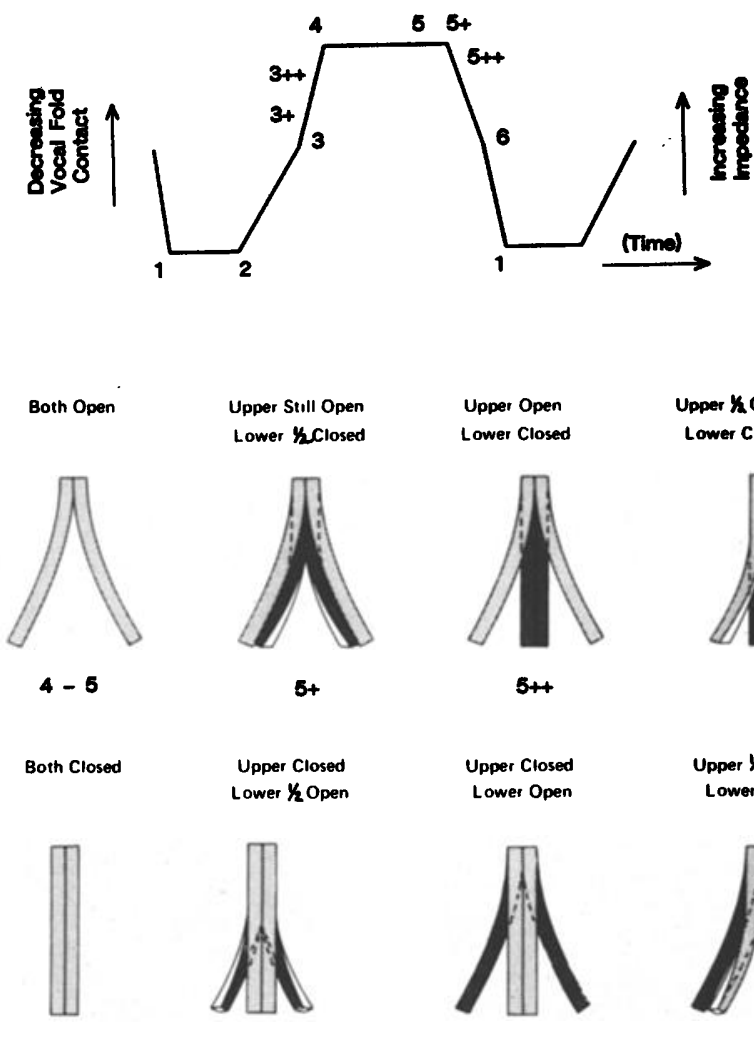

Upper K Closed Lower Closed

$1-2$

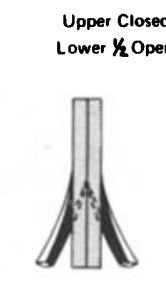

3

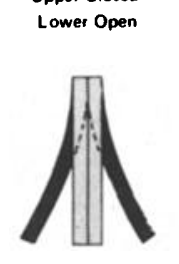
Lower Open

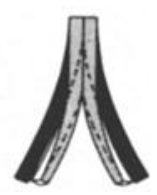

3+

3+1

FIG. 14. EGG waveform and an elastic two-mass vocal fold model. The vocal fold model events are labeled on the EGG waveform. The upper and lower vocal fold margins for each mass are out of phase. An artistic license was taken to illustrate vocal fold motion.

ment would be the incorporation of our model into the Flanagan-Ishizaka speech synthesis model. We should also be able to accommodate less restrictive geometric constraints on the vocal fold vibratory patterns.

Despite the highly speculative nature of these theoretical considerations, we are encouraged by the possible clinical application of the electroglottogram. The process of differential diagnosis of vocal pathologies has too long relied on purely subjective, qualitative assessment, which is solely observer dependent. As the move toward quantification of laryngeal behavior continues to grow in the health-related professions, the need for simple, reliable, low-cost instrumentation will increase. Our results encourage us to believe that the EGG may have a place in this process. For example, a set of EGG templates might be generated for a series of opening and closing vocal fold angles and lags. Then, one could compare a measured EGG waveform to these templates in an EGG atlas to infer aspects of vocal fold physiology, e.g., the manner in which the folds separate and collide. A similar atlas could be prepared for vocal fold nodules or polyps. A computer model, such as in Titze (1984), could also be developed to best estimate parameters of vocal fold vibration from measured EGG waveforms. Despite the obvious weaknesses of the model, the evidence we have presented is convincing that the EGG is related to the vocal fold contact area. Future work should substantiate this hypothesis even further. 


\section{ACKNOWLEDGMENTS}

This research was supported in part by NIH grant NS17078, NSF grant ECE-8413583, and the University of Flórida, College of Engineering Center of Excellence Program for our Mind-Machine Interaction Research Center.

Baer, T., Lofqvist, A., and McGarr, N. S. (1983a). "Laryngeal vibrations: A comparison between high-speed filming and glottographic techniques," J. Acoust. Soc. Am. 73, 1304-1308.

Baer, T., Titze, I., and Yoshioka, H. (1983b). "Multiple simultaneous measures of vocal fold activity," in Vocal Fold Physiology: Contemporary Research and Clinical Issues, edited by D. M. Bless and J. H. Abbs (College Hill, San Diego), pp. 229-237.

Bocchieri, E. L., and Childers, D. G. (1984). "Interactive graphics editor permits study of animated speech articulation," Speech Tech. 2, 10-14.

Childers, D. G., and Krishnamurthy, A. K. (1985). "A critical review of electroglottography," CRC Crit. Rev. Biomed. Eng. 12, 131-161.

Childers, D. G., Smith, A. M., and Moore, G. P. (1984). "Relationships between electroglottograph, speech, and vocal cord contact," Folia Phoniatr. 36, 105-118.

Childers, D. G., Naik, J. M., Larar, J. N., Krishnamurthy, A. K., and Moore, G. P. (1983). "Electroglottography, speech, and ultra-high speed cinematography," in Vocal Fold Physiology and Biophysics of Voice, edited by I. R. Titze and R. Scherer (Denver Center for the Performing Arts, Denver), Chap. 17, pp. 202-220.

Childers, D. G., Moore, G. P., Naik, J. M., Larar, J. N., and Krishnamurthy, A. K. (1982). "Assessment of laryngeal function by simultaneous, synchronized measurement of speech, electroglottography and ultra-high speed film," in Transcripts of the Eleventh Symposium Care of the Professional Voice (Juilliard School, New York), Vol. II, Medical/ Surgical Sessions: Papers, edited by L. Van Lawrence, The Voice Foundation, pp. 234-244.
Flanagan, J. L., and Ishizaka, K. L. (1976). "Automatic generation of voiceless excitation in a vocal cord, vocal tract speech synthesizer," IEEE Trans. Acoust. Speech Signal Process. ASSP-24, 163-169.

Flanagan, J. L., and Ishizaka, K. L. (1978). "Computer model to characterize the air volume displaced by the vibrating vocal cords," J. Acoust. Soc. Am. 63, 1559-1566.

Flanagan, J. L., and Ishizaka, K. L. (1975). "Synthesis of speech from a dynamical model of the vocal cords and vocal tract," Bell System Tech. J. 54, 484-506.

Flanagan, J. L., Ishizaka, K., and Shipley, K. L. (1980). "Signal model for low bit rate coding of speech," J. Acoust. Soc. Am. 68, 780-791.

Fourcin, A. J. (1981). "Laryngographic assessment of phonatory function," in Proceedings of the Conference on the Assessment of Vocal Pathology, ASHA Rep. No. 11, edited by C. L. Ludlow and M. O. Hart (American Speech and Hearing Association, Washington, D.C.), pp. 116-127.

Gilbert, H. R., Potter, C. R., and Hoodin, R. (1984). "Laryngograph as a measure of vocal fold contact area," J. Speech Hear. Res. 27, 173-178.

Ishizaka, K. L., and Flanagan, J. L. (1972). "Synthesis of voiced speech from a two mass model of the vocal cords," Bell System Tech. J. 51, 12331268.

Rothenberg, M. (1981). "Some relations between glottal air flow and vocal fold contact area," in Proceedings of the Conference on the Assessment of Vocal Pathology, ASHA Rep. No. 11, edited by C. L. Ludlow and M. O. Hart (American Speech and Hearing Association, Washington, D. C.), pp. 88-96.

Titze, I. R. (1984). "Parameterization of the glottal area, glottal flow, and vocal fold contact area," J. Acoust. Soc. Am. 75, 570-580.

Titze, I. R., Baer, T., Cooper, D., and Scherer, R. (1983). “Automated extraction of glottographic waveform parameters and regression to acoustic and physiologic variables," in Vocal Fold Physiology: Contemporary Research and Clinical Issues, edited by D. M. Bless and J. H. Abbs (College Hill, San Diego), pp. 146-154.

Titze, I. R., and Talkin, D. (1981) . "Simulation and interpretation of glottographic waveforms," in Proceedings of the Conference on the Assessment of Vocal Pathology, ASHA Rep. No. 11, edited by C. L. Ludlow and M. O. Hart (American Speech and Hearing Association, Washington, D. C.), pp. 48-55. 